\title{
Adiciones y Comentarios a la Flora de Honduras
}

\author{
Cyril H. Nelson ${ }^{1}$
}

Resumen. Se agregan y comentan taxones de la flora de Honduras, de los cuales hay 10 familias nuevas, 52 géneros, 338 especies, nueve subespecies, cinco variedades, una forma, 19 endemismos, 53 sinonimias y 28 nombres comunes.

Palabras clave: Familias, géneros, sinonimia, endemismo, nuevos registros, nombres comunes.

\section{Adiciones y Comentarios a la Flora de Honduras}

\begin{abstract}
Taxa of the flora of Honduras, of which there are 10 new families, 52 genera, 338 species, nine subspecies, five varieties, one form, 19 endemisms, 53 synonyms, and 28 common names are added and commented.
\end{abstract}

Kew words: Families, genera, synonymy, endemism, new records, common names.

\section{Introducción}

Desde la publicación de Nelson Sutherland (2008) del Catálogo de las Plantas Vasculares de Honduras han aparecido nuevos registros para la flora de Honduras, ya sea por la publicación de especies nuevas, ya sea por la corrección de especímenes mal identificados, ya sea por la identificación de antiguos especímenes que reposaban en herbarios esperando que un especialista los identificara. También la publicación del grupo APG III (2009) ha contribuido al reordenamiento de órdenes y familias en las plantas con flores.

Una de las principales fuentes para encontrar especímenes identificados, y que constituyen registros nuevos para la flora hondureña, es la base de datos $\mathrm{W}^{3}$ Tropicos del Jardín Botánico de Missouri de la ciudad de San Luis, Estado de Missouri, Estados Unidos de América. Algunas especies han pasado de sinonimia a nombres aceptados y viceversa.

\section{Discusión}

Los especímenes mencionados en Observación corresponden a ejemplos de plantas colectadas en Honduras solamente; van acompañados, salvo pocas excepciones, de los acrónimos de los herbarios donde están depositados, aunque hay que advertir que pudieran existir duplicados en otras instituciones. En el herbario del Jardín Botánico de Missouri (MO) es donde están depositadas la mayoría de las especies citadas. Sin embargo, no necesariamente es el único herbario donde hay duplicados, pudiéndose encontrar también en el herbario de la Escuela Agrícola Panamericana (EAP). La Flora Mesomericana (Davidse et al., 1994-) aún no completada, es una referencia para saber la aceptación o no de un taxon.

\section{Resultados}

A continuación se presenta un listado de adiciones y comentarios para la flora de Honduras por orden alfabético de familias y géneros. Sirve de punto de referencia básica la obra de Nelson Sutherland (2008).

Los nombres marcados con un asterisco $\left(^{*}\right)$ indican nuevos registros para la flora de Honduras; los marcados con dos asteriscos (**) son plantas citadas previamente como sinónimos, pero que ahora son aceptados.

\section{Acanthaceae}

**Ruellia blechum L., Syst. nat., ed. 10, 2:1120. 1759. Observación: C. Nelson, Ana L. Díaz, R. Rodríguez, R. Andino, H.A. Martínez \& E. Romero 7657 (MO, TEFH). Citada en Nelson Sutherland (2008) como Blechum pyramidatum (Plum. ex Lam.) Urb.

\footnotetext{
${ }^{1}$ Profesor jubilado, Departamento de Biología, Universidad Nacional Autónoma de Honduras, Tegucigalpa, Honduras, correo electrónico chnelsons@yahoo.com

DOI:10.5377/ceiba.v51i2.1105 
**Ruellia latibracteata D.N. Gibson, Fieldiana, Bot. 34(6):79. 1972. Observación: A. Molina R. 5119 (F). Citada en Nelson Sutherland (2008) como sinónimo de Blechum grandiflorum Oerst.

\section{Achariaceae}

*Chiangiodendron mexicanum $\mathrm{T}$. Wendt, Syst. Bot. 13(3):435, fig. 1-3, 4A, 5. 1988. Observación: Doblado Amador (2011) cita especie para el litoral atlántico de Honduras. No confundir este género con el género Changiodendron R.H. Miao (Sabiaceae).

\section{Achatocarpaceae}

*Achatocarpus pubescens C.H. Wright, Bull. Misc. Inform. Kew 1906:6. 1906. Observación: G. Davidse, M. Sousa \& J.L. Linares 35497 (MO), B.K. Holst 1784 (MO), 1818 (MO).

\section{Actinidiaceae}

*Saurauia homotricha A. Pool, Novon 8(3):280, fig. 1 (1998). Observación: A. Molina R. 6513 (US), G.E. Pilz \& M. Pilz 1354-b (MO), T.G. Yuncker 6261 (MO).

**Saurauia yasicae Loes., Bot. Jahrb. Syst. 23(1-2):125. 1896. Observación: J.M. MacDougal, P.R. House \& R. Zúñiga 3365 (MO), C. Nelson, E. Vargas \& M. Erazo 3110 (MO, TEFH). Citado en Nelson Sutherland (2008) como Saurauia laevigata Triana \& Planch.

\section{Amaranthaceae}

**Iresine herbstii Hook., Gard. chron. 1864:654. 1864. Observación: A. Molina R. 34050 (MO). Citado en Nelson Sutherland (2008) como Iresine diffusa Humb. \& Bonpl. ex Willd. fma. herbstii (Hook.) Pedersen.

\section{Amaryllidaceae}

*Hymenocallis latifolia (Mill.) M. Roem., Fam. Nat. Syn. Monogr. 4:168. 1847. Observación: G.R. Proctor 32562 (IJ).

\section{Anacardiaceae}

**Amphipterygium simplicifolium (Standl.) Cuev.-Fig., Ibugana 13(1):40, fig. 1-2, 13-15, 20. 2005. Observación: Previamente identificada como Amphipterygium adstringens (Schltdl.) Standl.

\section{Annonaceae}

**Annona mucosa Jacq., Fl. Bras. Merid., ed. quarto, 1:23. 1824. Observación: D.L. Hazlett, A. Brant \& R. Zúñiga 8007 (MO). Citada en Nelson Sutherland (2008) como sinónimo de Rollinia mucosa (Jacq.) Baill.

*Annona subnubila H. Rainer, Inédito. Hermes Vega (foto). *Cymbopetalum torulosum G.E. Schatz, Ann. Missouri Bot. Gard. 72(3):535, fig. 1-2, 4, 7-8. 1985. Observación: $R$. Zúñiga 428 (TEFH).

**Guatteria tomentosa Rusby, Bull. New York Bot. Gard. 6:504. 1910. Observación: A. Brant \& R. Zúñiga 2814 (MO), T. Hawkins \& M. Merello 796 (MO), P.J.M. Maas, H. Maas, R. Zúñiga \& T. Gómez 8496 (MO), D.L. Hazlett 3333 (MO). Observación: Registrada como Guatteria dolichopoda Donn. Sm. en Nelson Sutherland (2008).

*Ruizodendron ovale R.E. Fr., Ark. Bot. 28B:3. 1936. Observación: C. Nelson 818 (MO, TEFH). Primer registro de este género y especie para Mesoamérica.

*Sapranthus hirsutus G.E. Schatz, Inédito. Observación: A. Molina R. 13657 (F), L.O. Williams \& A. Molina R. 188053 (MO), holotipo.

*Stenanona hondurensis G.E. Schatz, F.G. Coe \& Maas in G.E.Schatz \& Maas, Blumea 55(3): 211, tab. 1, mapa. 2010. Observación: A. Brant \& R. Zúñiga 2840 (MO), tipo; isotipos (CR, EAP, INB, K, PMA, STRI, WAG). Otras colecciones, R. Aguilar 4062 (MO?), 4088 (MO). Schatz y Maas (2010) publican esta especie como endémica de Honduras.

*Stenanona tuberculata G.E. Schatz \& Maas, Blumea 55(3):219, tab. 4, mapa 2. 2010. Observación: T. Hawkins \& M. Merello 743 (MO), tipo; isotipos (EAP, F, US, WAG), A. Brant \& R. Zúñiga 2832 (MO), D. Hazlett, A. Brant \& R. Zúñiga 8030 (¿?). Schatz y Maas (2010) publican esta especie como endémica de Honduras.

\section{Apocynaceae}

*Echites puntarenensis J.F. Morales, Brittonia 49(3):332. 1997. Observación: A. Molina R. 485 (EAP).

*Couma macrocarpa Barb. Rodr., Vellosia, ed.2, 1:32, tab. 1, fig. b. 1891. Observación: E. Vargas et al. 382 (MO, TEFH?). Nombres comunes: palo de leche, palo de vaca.

*Gonolobus versicolor Woodson, Publ. Field Mus. Nat. Hist., Bot. Ser. 23(2):80. 1944. Observación: A.F. Clewell \& D.L. Hazlett 3862 (MO).

*Gonolobus xanthotrichus Brandegee, Zoё 5(11):251. 1908. Observación: L.O. Williams \& A. Molina R. 18078 (DS, EAP, F). 
*Macroscepis hirsuta (Vahl) Schltr., Symb. antill. 1(2):265. 1899. Observación: A. Molina R. 5256 (F).

*Marsdenia gilgiana W. Rothe, Bot. Jahrb. Syst. 52:410. 1915. Observación: A. Molina R. 157 (F).

*Marsdenia nicoyana Pittier, Contr. U.S. Natl. Herb. 13(4):102. 1910. Observación: B.K. Holst 1083 (TEFH), A. Molina R. 3938 (F), A. Molina R. \& A.R. Molina 13824 (F).

**Matelea warscewiczii (H. Karst.) Woodson, Ann. Missouri Bot. Gard. 28(2):224. 1941. Observación: A. Molina R. 2561 (F), A. Molina R. 3209 (EAP, F). Citado en Nelson Sutherland (2008) como Matelea marsdenioides Standl. \& L.O. Williams en la familia de las Asclepiadaceae. **Metastelma trichophyllum (L.O. Williams) W.D. Stevens, Phytologia 64(5):335. 1988. Observación: $M$. Espinal 188 (MO, TEFH). Citado en Nelson Sutherland (2008) como Metastelma schlechtendalii Decne. var. trichophylum (L.O. Williams) Liede \& Meve.

*Orthosia ramosa W.D. Stevens, Novon 15(4):637, tab. 9. 2005. Observación: A. Molina R. \& A.R. Molina 30910 (MO), tipo; isotipos (EAP, F), G. Davidse \& R. Zúñiga 34697 (MO). Stevens (2005) publican esta especie como endémica de Honduras colectado en el departamento de Ocotepeque.

**Tabernaemontana odontadeniiflora A.O. Simões \& M.E. Endress, Taxon 59(3):78. 2010. Observación: $C$. Hughes 821 (MO). Nombre nuevo para Stemmadenia pubescens Benth., nombre con el cual es citado en Nelson Sutherland (2008).

*Thenardia chiapensis J.K. Williams, Brittonia 47(4):403, fig. 1-3. 1995. Observación: R. Keyser 1163 (MO).

\section{Araceae}

*Epipremnum pinnatum (L.) Engler, Pflanzenr. IV. 23B(Heft 37): 60. 1908. Observación: fotografiada por Cenaida Moncada en el Jardín Botánico de Lancetilla, Tela, Departamento de Atlántida, Honduras.

*Monstera maderaverde Grayum \& Karney, Econ. Bot. 66(2):209, fig. 3-5. 2012.

Observación: A. Karney s.n. (MO-6240501 (tipo); isotipo (CURLA). Grayum y Karney (2012) publican esta especie como endémica de Honduras.

*Syngonium salvadorense Schott, Oesterr. Bot. Z. 8(6):178. 1858. Observación: A. Molina R. 33722 (MO).

*Urospatha grandis Schott, Bonplandia (Hannover) 5(8):128. 1857. Observación: fotografiada por Josué Ramos Galdámez en los alrededores de La Ceiba, Departamento de Atlántida, Honduras.

\section{Araliaceae}

**Oreopanax guatemalensis (Lem. ex Bosse) Decne. \& Planch., Rev. Hort. 16:108. 1854. Observación: A. Molina R. 30788 (F). Citado en Nelson Sutherland (2008) como sinónimo de Oreopanax obtusifolius L.O. Williams.

*Polyscias paniculata (DC.) Baker, Fl. Mauritius: 126. 1877. Observación: A. Molina R. \& A.R. Molina 34940 (MO).

\section{Arecaceae}

*Calyptrogyne ghiesbreghtiana (Linden \& H. Wendl.) H. Wendl. subsp. hondurensis A.J. Hend., Syst. Bot. 30(1):81, fig. 5F, mapa. 2005. Observación: G. Davidse, R. Zúñiga \& P.R. House 34399 (NY), tipo; isotipos (MO, TEFH). Henderson (2005) describe esta subespecie como endémica de Honduras.

*Chamaedorea rojasiana Standl. \& Steyerm., Publ. Field Mus. Nat. Hist., Bot. Ser. 23(5):205. 1947. Observación: R.J. Evans 2562 (MO).

**Geonoma pinnatifrons Willd. subsp. mexicana (Liebm. ex Mart.) A.J. Hend., Phytotaxa 17:121, fig. 32. 2011. Observación: R.J. Evans, M.H. Grayum \& A. Chinchilla 2747 (MO). Citada en Nelson Sutherland (2008) como sinónimo de Geonoma oxycarpa Plum. ex Mart.

**Geonoma undata Klotszch subsp. edulis (H. Wendl. ex Spruce) A.J. Hend., Phytotaxa 17:158, fig. 42. 2011. Observación: T. Hawkins 492 (MO), R.J. Evans 1459 (MO), 1741 (MO). Citada en Nelson Sutherland (2008) como sinónimo de Geonoma interrupta (Ruiz \& Pav.) Mart.

\section{Aristolochiaceae}

*Aristolochia constricta Griseb., Abh. Königl. Ges. Wiss. Göttingen 7:225. 1857. Observación: D.L. Hazlett \& A. Brant 8048 (MO).

\section{Asteraceae (Compositae)}

*Ageratina rivalis (Greenm.) R.M. King \& H. Rob., Phytologia 19(4):216. 1970. Observación: C. Nelson, J.R. Martínez \& J. Espinoza 1651 (MO, TEFH).

*Ageratum maritimum Kunth, Nov. gen. sp., ed. folio, 4:117. 1818 [1820]. Observación: C. Nelson \& E. Romero 4509 (MO, TEFH).

*Anthemis cotula L., Sp. pl. 2:894. 1753. Observación: A. Molina R. 15233 (NY). 
**Baccharis lancifolia Less., Linnaea 9:266. 1834. Observación: C. Nelson, J.R. Martínez \& J. Espinoza 1593 (MO, TEFH). Citada en Nelson Sutherland (2008) como sinónimo de Baccharis vaccinioides Kunth.

*Baccharis salicifolia (Ruiz \& Pav.) Pers. subsp. monoica (G.L. Nesom) Joch. Müll., Syst. Bot. Monogr. 76:306. 2006. Observación: L.O. Williams \& A. Molina R. 10514 (MO).

**Chloracantha spinosa (Benth.) G.L. Nesom, Phytologia 70(5):378. 1991. Observación: C. Nelson, M. Hernández \& A. Rosales 202 (MO, TEFH). Citada en Nelson Sutherland (2008) como Aster spinosus Benth.

*Chrysanthemum morifolium Ramat., J. Hist. Nat. 2:240. 1792. Observación: A. Molina R. \& A.R. Molina 35142 (MO).

*Eupatorium hospitale B.L. Rob., Proc. Amer. Acad. Arts 43:32. 1907. Observación: C. Nelson \& E. Vargas 2438 (MO, TEFH).

*Gnaphalium greenmanii S.F. Blake, J. Wash. Acad. Sci. 21(14):329. 1931. Observación: B.K. Holst 1582 (MO).

*Mikania cordata (Burm. f.) B.L. Rob., Contr. Gray Herb. 104:65. 1934. Observación: C. Nelson, E. Romero, R. Andino, H.A. Martínez, A.L. Díaz \& R. Rodríguez 7378 (MO, TEFH).

*Psacaliopsis pinetorum (Hemsl.) Funston \& Villaseñor, Ann. Missouri Bot. Gard. 95(2):334. 2008. Observación: A. Molina R. 22122 (F).

**Pseudogynoxys haenkei (DC.) Cabrera, Brittonia 7(2):54. 1950. Observación: A. Molina R. 732 (MO), 30044 (MO). Citada como Pseudogynoxys chenopodioides (Kunth) Cabrera en Nelson Sutherland (2008).

*Sabazia liebmannii Klatt, Leopodina 23:90. 1887. Observación: A. Molina R. \& A.R. Molina 26090 (MO).

*Spiracantha cornifolia Kunth, Nov. gen. sp. ed. folio 4:23, tab. 313. 1818 [1820]. Observación: A. Molina R., A.R. Molina \& J.A. Molina 33459 (MO), 34399 (MO). Nombre común: mozote.

*Stevia rebaudiana (Bertoni) Bertoni, Anales Ci. Parag. 1(5):3. 1905. Observación: A. Molina R. \& A.R. Molina 35128 (MO), 35144 (MO).

*Verbesina lanata B.L. Rob. \& Greenm., Proc. Amer. Acad. Arts 34(20):558. 1899. Observación: D. Mejía (EAP, $\mathrm{MO}, \mathrm{TEFH})$.

\section{Basellaceae}

*Anredera ramosa (Moq.) Eliasson, Madroño 20(5):266. 1970. Observación: B.K. Holst 1380 (MO).

\section{Begoniaceae}

*Begonia lubbersii E. Morren, Belgique Hort. 33:155, tab. 13. 1883. Observación: A. Molina \& A. R. Molina 34289 (MO).

*Begonia pseudopeltata Burt-Utley \& Utley, Phytoneuron 2012(74):11, fig. 5. 2012. Observación: R.J. Evans 1154 (MO), tipo; isotipo (USF). Otra colección: P.R. House 892 (EAP, HEH, MO, TEFH, USF). Endémico de Guatemala y Honduras.

\section{Berberidaceae}

**Berberis hemsleyi Donn. Sm., Prim. fl. costaric. 2:17. 1898. Observación: PH. Allen, R. Armour \& A. Chable 6071 (US), tipo de Mahonia glauca Standl. \& L.O. Williams, transferido en 1956 del Herbario EAP al Herbario Nacional de Estados Unidos (US) por L.O. Williams (Dorr et al. 2009). Citado en Nelson Sutherland (2008) como Berberis standleyi Marroq. \& Laferrière.

\section{Bignoniaceae}

*Adenocalymma inundatum Mart. ex DC., Prodr. 9:201. 1845. Observación: A. Molina R. \& A.R. Molina 24710 (F).

*Arrabidaea viscida (Donn. Sm.) A.H. Gentry, Wrightia 7(2):84. 1982. Observación: L.O. Williams \& A. Molina R. s.n. $(\mathrm{F})$.

\section{Boraginaceae}

**Bourreria succulenta Jacq., Enum. syst. pl.:14. 1760. Observación: C. Nelson, D. Ruiz \& S. Gómez 9698 (MO, TEFH), G.R. Proctor 32489 (BM, F, LL). Citada en Nelson Sutherland (2008) como Bourreria baccata Raf.

\section{Brassicaceae (Cruciferae)}

*Cardamine ovata Benth., Pl. hartw.: 158. 1845. Observación: A.F. Clewell \& D.L. Hazlett 3955 (MO).

*Lepidium costaricense Thell., Bull. Herb. Boissier, sér. 2, 4(7):713. 1904. Observación: A. Molina R., A.R. Molina \& J.A. Molina 33530 (MO). Nombre común: mastuerzo.

**Lepidium didymum L., Syst. nat. ed. 12, 2:433. 1767; vide Mant. pl.:92. 1767. Observación: A. Molina R. 18378 (DS). Citado en Nelson Sutherland (2008) como Coronopus didymus (L.) Sm. 


\section{Bromeliaceae}

*Pitcairnia saxicola L.B. Sm., Contr. Gray Herb. 117:29. 1937. Observación: T. Hawkins 626 (EAP, MO).

\section{Buxaceae}

**Haptanthus hazlettii Goldberg \& C. Nelson, Syst. Bot. 14(1):16, fig. 1-3. 1989. Observación: Esta planta estaba en la familia Haptanthaceae, pero estudios recientes de ADN de Shipunov y Shipunova (2011) sugieren que queda mejor ubicada en la familia Buxaceae, lo que constituye una nueva familia para la flora de Honduras.

\section{Cactaceae}

*Schlumbergera gaertneri (Regel) E. Britton \& A. Rose, Contr. U. S. Natl. Herb. 16(9):260. 1913. Observación: A. Molina R. 33893 (MO). Nombre común: estrella de Navidad.

\section{Capparaceae}

*Capparidastrum pachaca (Kunth) Hutch. subsp. oxysepalum (C. Wright ex Radlk.) Iltis, Harvard Pap. Bot. 13(2):235. 2008. Observación: B.K. Holst 1711 (MO). Nombre común: palo de rosa.

*Quadrella lundellii (Standl.) Iltis \& Cornejo, J. Bot. Res. Inst. Texas 4(1):127. 2010. Observación: P.R. House s.n. (TEFH).

\section{Caryophyllaceae}

*Cerastium fontanum Baumg. subsp. vulgare (Hartm.) Greuter \& Burdet, Willdenowia 12(1):37. 1982. Observación: L.O. Williams \& A. Molina R. 17091 (MO).

\section{Celastraceae}

**Cuervea kappleriana (Miq.) A.C. Sm., Brittonia 3(3):399. 1940. Observación: C. Thieme 5343 (US). Citada en Nelson Sutherland (2008) como Cuervea mitchellae (I.M. Johnst.) A.C. Sm., la cual en Davidse et al. (1994-) es considerada como sinónimo de Cuervea kappleriana (Miq.) A.C. Sm.

\section{Cleomaceae}

Podandrogyne pulcherrima (Standl.) Cochrane, Novon (inédito). Observación: T. Hawkins \& D. Mejía 235 (MO, WIS).
*Tarenaya parviflora (Kunth) Iltis, Inédito. Observación: D. Burch 5481 (MO), C. Thieme 5134 (GH).

\section{Clusiaceae}

*Clusia ovigera Triana \& Planch., Ann. Sci. Nat., Bot. 13:354. 1860. Observación: T. Hawkins 494 (MO).

\section{Combretaceae}

*Combretum rovirosae Exell, J. Linn. Soc. Bot. 55(356):134, fig. 5e. 1953. Observación: C. Nelson \& J.R. Martínez 1148 (MO, TEFH).

**Terminalia buceras (L.) C. Wright, Anales Acad. Ci. Med. Habana 5:409. 1869. Observación: A.H. Gentry 7595 (MO), C. Nelson, E. Vargas \& M. Erazo 3169 (MO, TEFH), G. Padilla 40 (MO), G. Soihet 206 (MO). Citada en Nelson Sutherland (2008) como Bucida buceras L.

**Terminalia macrostachya (Standl.) Stace, (Inédito). Observación: G. Davidse, M. Sousa \& J.L. Linares 35044 (MO), D.L. Hazlett 2019 (MO). Citada en Nelson Sutherland (2008) como Bucida macrostachya Standl.

\section{Convolvulaceae}

**Ipomoea bernoulliana Peter, Nat. Pflanzenfam. IV (3A):30. 1891 [1897]. Observación: C. Nelson 3924 (MO, TEFH), 7407 (MO, TEFH). Citada en Nelson Sutherland (2008) como Ipomoea santae-rosae Standl. \& Steyerm.

*Ipomoea x leucantha Jacq., Icon. pl. rar. 2:10, tab. 318. 1788. Observación: R.J. Evans 1306 (MO).

*Ipomoea mairetii Choisy, Prodr. 9:374. 1845. Observación: A. Molina R. 3493 (MO), A. Molina R. \& A.R. Molina 34235 (MO). Nombre común: cuerda de Nazareno. *Ipomoea villifera House, Muhlenbergia 5(5):70. 1909. Observación: A. Molina R., A.R. Molina \& J.A. Molina 31434 (MO).

\section{Cordiaceae}

**Varronia bullata L., Syst. nat. ed. 10, 2:916. 1759. Observación: C. Nelson 2999 (MO, TEFH). Citado en Nelson Sutherland (2008) como sinónimo de Cordia bullata (L.) Roem. \& Schult.

**Varronia curassavica Jacq., Enum. syst. pl.:14. 1760. Observación: A. Molina R. 951 (MO). Citado en Nelson Sutherland (2008) como sinónimo de Cordia curassavica (Jacq.) Roem. \& Schult.

*Cordia cymosa (Donn. Sm.) Standl., Publ. Field Mus. Nat. Hist., Bot. Ser. 18(3): 981. 1938. Observación: T. Hawkins \& M. Merello 788 (MO). 
**Varronia globosa Jacq., Enum. syst. pl.:14. 1760. Observación: A. Molina R. 14369 (F). Citado en Nelson Sutherland (2008) como sinónimo de Cordia globosa (Jacq.) Kunth.

*Varronia guanacastensis (Standl.) J.S. Mill., Novon 17(3):373. 2007. Observación: A. Molina R. 1062 (MO), G.L. Webster 12004 (F).

*Varronia inermis (Mill.) Borhidi, Acta Bot. Hung. 34(34):389. 1988. Observación: C. Nelson 4793 (MO, TEFH).

**Varronia linnaei (Stearn) J.S. Mill., Novon 17(3):374. 2007. Observación: C. Nelson et al. 5979 (MO, TEFH). Citado en Nelson Sutherland (2008) como Cordia linnaei Stearn.

*Varronia macrocephala Desv., J. Bot. (Desvaux) 1:273. 1808. Observación: A. Molina R. 13025 (US).

**Varronia spinescens (L.) Borhidi, Acta Bot. Hung. 34(34):393. 1988. Observación: T.G. Yuncker 4572 (MO). Citada en Nelson Sutherland (2008) como Cordia spinescens L.

\section{Crassulaceae}

*Kalanchoe mortagei Hamet \& H. Perrier, Bull. Soc. Bot. France 95:120. 1948. Observación: A. Molina R. \& A.R. Molina 34701 (MO).

\section{Cupressaceae}

**Cupressus corneyana Carrière, Traité gen. conif.:128. 1855. Observación: Citada en Nelson Sutherland (2008) como Cupressus funebris Endl.

\section{Cyclanthaceae}

**Asplundia utilis (Oerst.) Harling, Acta Horti Berg. 17(3):43. 1954. Observación: R. Aguilar \& R.J. Evans 4075 (MO). Citada como sinónimo de Asplundia costaricensis Harling p.p. en Nelson Sutherland (2008).

\section{Cyperaceae}

*Fuirena scirpoidea Michx., Fl. bor.-amer. 1:37, tab. 7. 1803. Observación: S. Fawcett \& T. Phillips SF2011.

* Rhynchospora tracyi Britton, Trans. New York Acad. Sci. 11(3-5):84. 1892. Observación: S. Fawcett, T. Phillips \& A. Rebertus SF0211.

\section{Ericaceae}

*Vaccinium lundellianum L.O. Williams, Fieldiana, Bot., 31(6):173. 1965. Observación: T. Hawkins 919 (MO).

\section{Euphorbiaceae}

**Alchornea triplinervia (Spreng.) Müll. Arg., Prodr. 15(2):909. 1866. Observación: Citada en Nelson Sutherland (2008) como Alchornea obovata Pax \& K. Hoffm.

**Argythamnia ecdyomena J.W. Ingram, Gentes Herb. 10(1):16, fig. 9B. 1967. Observación: C. Nelson \& E. Romero 4573 (MO, TEFH). Citada en Nelson Sutherland (2008) como Argythamnia tinctoria Millsp.

**Astraea lobata (L.) Klotzsch, Arch. Naturgesch. 7(1): 194. 1841. Observación: C. Nelson \& E. Romero 4429 (MO, TEFH). Citada en Nelson Sutherland (2008) como Croton lobatus L.

*Bernardia dodecandra (Sessé ex Cavagnaro, David) McVaugh, Bot. Results Sessé \& Mociño (1787-1803): 222. 2000. Observación: B.K. Holst 1302 (MO).

**Croton nitens Sw., Prodr.: 100. 1788. Observación: $C$. Nelson, D. Ruiz \& S. Gómez 9758 (MO, TEFH). Citada en Nelson Sutherland (2008) como sinónimo de Croton glabellus L.

*Euphorbia cotinifolia L. subsp. cotinoides (Miq.) Christenh., Harvard Pap. Bot. 7(1):3. 2002. Observación: A. Molina R. \& A.R. Molina 31483 (MO).

\section{Fabaceae}

*Abrus precatorius L. subsp. africanus Verdc., Kew Bull. 24(2):241. 1970. Observación: A.F. Clewell 4018 (MO, TEFH), C. Nelson \& M. Hernández 165 (MO, TEFH), J.G. Saunders 537 (MO, TEFH). Naturalizada originaria de Kenia.

**Acacia viminalis Aiton ex Steud., Nomencl. bot., ed. 2, 1:8. 1840. Citado en Nelson Sutherland (2008) como sinónimo de Acacia retinodes Schltdl.

*Caesalpinia decapetala (Roth) Alston, Handb. fl. Ceylon 6:89. 1931. Observación: A. Molina R. 33889 (MO).

Cultivada.

**Caesalpinia yucatanensis Greenm., Publ. Field Columb. Mus., Bot. Ser. 2(6):252. 1907. Observación: Citada en Nelson Sutherland (2008) como Caesalpinia recordii Britton \& Rose.

*Calliandra bijuga Rose, Contr. U.S. Natl. Herb. 5(3):135. 1897. Observación: M. Sousa, G. Davidse \& J.L. Linares 13297 (MO).

*Centrosema molle Mart. ex Benth., Comm. legum. gen.:55. 1837. Observación: A. Molina R. \& A.R. Molina 24655 (MO).

*Centrosema schottii (Millsp.) K. Schum., Just's Bot. Jahresber. 26(1):353. 1900. Observación: A. Molina R., A.R. Molina \& J.A. Molina 32125 (MO). 
*Coursetia glandulosa A. Gray, Proc. Amer. Acad. Arts 5:156. 1861. Observación: J.R. Martínez 12 (MO).

*Dalbergia chontalensis Standl. \& L.O. Williams, Ceiba 1(2):81. 1950. Observación: A. Molina R. 1526 (MO).

*Desmodium heterocarpon (L.) DC., Prodr. 2:337. 1825. Observación: A. Molina R. 34445 (MO).

**Leptolobium panamense (Benth.) Sch. Rodr. \& A.M.G. Azevedo, Taxon 57(3):983. 2008. Observación: C. Nelson, E. Romero, H.A. Martínez, R. Andino, R. Rodríguez \& A.L. Díaz 5745 (MO, TEFH). Citada en Nelson Sutherland (2008) como sinónimo de Acosmium panamense Benth.

*Lonchocarpus lanceolatus Benth. subsp. calciphilus M. Sousa, Acta Bot. Mex. 86:46, fig. 1. 2009. Observación: $M$. Sousa \& O. Téllez V. 8677 (MEXU), holotipo. Sousa Sánchez (2009a) menciona la presencia de este taxon en Honduras en los departamentos de Choluteca y Francisco Morazán.

*Lonchocarpus parviflorus Benth., J. Proc. Linn. Soc., Bot. 4. Suppl.: 89. 1860. Observación: M. Sousa, G. Davidse \& J.L. Linares 13299 (MO).

*Lonchocarpus purpureus Pittier, Contr. U. S. Natl. Herb. 20(2):65, fig. 13. 1917. Observación: M. Sousa, G. Davidse \& J.L. Linares $13344(\mathrm{MO})$.

**Lonchocarpus rugosus Benth. subsp. apricus (Lundell) M. Sousa, Fl. Nova-Galiciana 5:573. 1987. Observación: $C$. Nelson 203 (MEXU, TEFH). Citada en Nelson Sutherland (2008) como sinónimo de Lonchocarpus rugosus Benth. Nombres comunes: cincho, coyote y quebracho en Sosa Sánchez (2008).

*Lonchocarpus rugosus Benth. subsp. gillyi (Lundell) M. Sousa, Ceiba 49(1): 126. 2008. Observación: C. Nelson 7179 (MEXU, MO, TEFH). Nombre común: masicarán en Sousa Sánchez (2008).

*Lonchocarpus semideserti M. Sousa, Acta Bot. Mex. 86:57, fig. 4. 2009. Observación: J.J. Hellin \& C.E. Hughes 5 (MEXU), holotipo, (EAP), isotipo. Sousa Sánchez (2009a) publica esta especie como endémica de México y Honduras. **Lonchocarpus vallicola (Standl. \& F.J. Herm.) M. Sousa, Novon 19(2):251, fig. 5. 2009. Observación: L.O. Williams \& A. Molina R. 11072 (F), holotipo. Standley \& Hermann en Hermann (1949) publican a Terua vallicola Standl. \& F.J. Herman, su basiónimo, como endémico de Honduras, lo mismo que Sousa Sánchez (2009b) cuando publica la nueva combinación.

**Lupinus mexicanus Cerv. ex Lag., Gen. sp. pl.:22. 1816. Observación: A. Molina R. 34030 (MO), A. Molina R. \& A.R. Molina 34843 (MO). Citada en Nelson Sutherland como Lupinus ehrenbergii Schltdl.

**Machaerium pittieri J.F. Macbride, Publ. Field Mus. Nat. Hist., Bot. Ser. 4(4):91. 1925. Observación: A. Molina R.
141 (MO), A. Molina R. \& A.R. Molina 24707 (MO). Citado en Nelson Sutherland (2008) como sinónimo de Machaerium arboreum (Jacq.) Vogel.

**Mariosousa centralis (Britton \& Rose) Seigler \& Ebinger, Novon 16(3):417. 2006. Observación: L.O. Williams \& A. Molina R. 10509 (MO). Citado en Nelson Sutherland (2008) como Acacia centralis (Britton \& Rose) Lundell.

**Mariosousa usumacintensis (Lundell) Seigler \& Ebinger, Novon 16(3):419. 2006. Observación: P.R. House \& E.Y. Guerrero 4972 (EAP, TEFH). Citada en Nelson Sutherland (2008) como Acacia usumacintensis Lundell.

* Mimosa bahamensis Benth., J. Bot. (Hooker) 4(32):408. 1842. Observación: C. Nelson, E. Vargas \& C. Alduvín 3367 (MO, TEFH). Nombres comunes: pelapija, rabo de iguana.

**Mimosa invisa Mart. ex Colla, Herb. Pedem. 2:255. 1834. Observación: A. Molina R., A.R. Molina \& J.A. Molina 33472 (MO). Citada en Nelson Sutherland (2008) como sinónimo de Mimosa diplotricha C. Wright ex Sauvalle var. diplotricha. Nombre común: rabo de iguana.

**Phaseolus leptostachyus Benth., Comm. legum. gen.:72. 1837. Observación: A. Molina R. 31749 (MO), L.O. Williams \& A. Molina R. 10555 (MO). Citada como sinónimo de Phaseolus anisotrichos Schltdl. Nombre común: chorequito.

**Pithecellobium oblongum Benth., London J. Bot. 3:198. 1844. Observación: A. Molina R. 33748 (MO). Citado en Nelson Sutherland (2008) como sinónimo de Pithecellobium unguis-cati (L.) Mart. Nombre común: mangollano.

*Psophocarpus tetragonolobus (L.) DC., Prodr. 2:403. 1825. Observación: A. Molina R. 33720 (MO). Nombre común: frijol alado.

**Senna pallida (Vahl) H.S. Irwin \& Barneby, Mem. New York Bot. Gard. 35(2):531. 1982. Observación: Citada en Nelson Sutherland (2008) como sinónimo de Senna galefigolia (L.) Barneby \& Lourteig.

**Swartzia guatemalensis (Donn. Sm.) Pittier, J. Wash. Acad. Sci. 11(7):159. 1921. Observación: citada como Swartzia myrtifolia Sm., D. Hazlett 3269 (MO), y Swartzia myrtifolia var. standleyi (Britton \& Rose) R.S. Cowan, G. Davidse 34369 (MO) en Nelson Sutherland (2008).

\section{Fagaceae}

**Quercus skinneri Benth., Pl. hartw.: 90. 1842. Observación: G. Davidse, M. Sousa \& J.L. Linares 35356 (MO), P.R. House 874 (EAP, MO, TEFH). Citada en Nelson Sutherland (2008) como sinónimo de Quercus xalapensis Humb. \& Bonpl. 


\section{Gentianaceae}

*Lisianthius cuspidatus Bertol., Novi Comment. Acad. Sci. Inst. Bononiensis 4:408. 1840. Observación: A. Molina R. \& A.R. Molina 31069 (F).

\section{Gesneriaceae}

*Amalophyllon macrophylloides Boggan \& L.E. Skog, Selbyana 29(2):165, fig. 2. 2008. Observación: G. Davidse et al. 34402 (US), tipo; isotipos (EAP, MO, TEFH), D.L. Hazlett, A. Brant \& R. Zúñiga 8019 (MO). Boggan (2008) la publica como endémica de Honduras.

**Amalophyllon repens (Donn. Sm.) Boggan, L.E. Skog \& Roalson, Selbyana 29(2):168. 2008. Observación: A. Molina R. \& A.R. Molina 31034 (MO). Citada en Nelson Sutherland (2008) como Phinaea repens (Donn. Sm.) Soler.

**Glossoloma cucullatum (C.V. Morton) J.L. Clark, Selbyana 25(2):200. 2005. Observación: Citada en Nelson Sutherland (2008) como Alloplectus cucullatus C.V. Morton.

**Glossoloma tetragonum Hanst., Linnaea 26:209. 1854. Observación: T.B. Croat \& D.P. Hannon 64250 (MO). Citada en Nelson Sutherland (2008) como sinónimo de Alloplectus tetragonus (Hanst.) Hanst.

*Kohleria tigridia (Ohlend.) Roalson \& Boggan, Selbyana 25(2):229. 2005. Observación: A. Molina R., L.O. Williams, A.R. Molina, T.P. Williams \& J.A. Molina 31322 (MO).

**Solenophora toucana D.L. Denham \& D.N. Gibson, Phytologia 23(4):341. 1972. Observación: A.F. Clewell \& D.L. Hazlett 3889 (MO). Citada como sinónimo de Solenophora calycosa Donn. Sm. en Nelson Sutherland (2008).

*Streptocarpus saxorum Engl., Bot. Jahrb. Syst. 19:154. 1894. Observación: A. Molina R., A.R. Molina \& J.A. Molina 34206 (MO).

\section{Goupiaceae}

**Goupia guatemalensis Lundell, Phytologia 57(3):238. 1985. Observación: C. Nelson \& G. Cruz 9475A (MO, TEFH). Citado en Nelson Sutherland (2008) como Goupia glabra Aubl.

\section{Gunneraceae}

**Gunnera mexicana Brandegee, Univ. Calif. Publ. Bot. 10(2):186, fig. 1 (1922). Observación: A. Molina R. 8218 (F). Citada en Nelson Sutherland (2008) en la familia de las Haloragaceae.

\section{Hamamelidaceae}

**Molinadendron guatemalense (Radlk. ex Harms) P.K. Endress, Bot. Jahrb. Syst. 89(3):357. 1969. Citada en Nelson Sutherland (2008) como Molinadendron hondurense (Standl.) P.K. Endress.

\section{Heliconiaceae}

**Heliconia mathiasiae G.S. Daniels \& F.G. Stiles, Brenesia 15. Supl.: 33, tab. 1, 3. 1979. Observación: A.F. Clewell 4653 (MO). Citado en Nelson Sutherland (2008) como Heliconia vaginalis Benth. subsp. mathiasiae (G.S. Daniels \& F.G. Stiles) L. Andersson.

\section{Heliotropiaceae}

**Tournefortia mutabilis Vent., Choix pl.: tab. 3 (1803). Observación: A. Molina R. 24633 (MO). Citada en Nelson Sutherland (2008) como sinónimo de Tournefortia densiflora M. Martens \& Galeotti. La familia Heliotropiaceae está formada por los géneros Heliotropium L. y Tournefortia L.

**Tournefortia umbellata Kunth, Nov. gen. sp. 3:79. 1818. Observación: T.B. Croat \& D.P. Hannon 64157 (MO), B.K. Holst 1678 (MO). La especie Tournefortia belizensis Lundell, citada en Nelson Sutherland (2008) es sinónimo de Tournefortia umbellata Kunth.

\section{Hydrangeaceae}

**Philadelphus mexicanus Schltdl., Linnaea 13:418. 1839. Observación: T. Clare 112 (MO), L. Tróchez 77 (MO). Citada en Nelson Sutherland (2008) como Philadelphus myrtoides Bertol.

\section{Hypericaceae}

*Hypericum silenoides Juss., Ann. Mus. Natl. Hist. Nat. 3:161, tab. 16, fig. 3. 1804. Observación: A. Molina R. 22141 (MO), A. Molina R. \& A. R. Molina 34840 (MO), 35040 (MO).

\section{Icacinaceae}

*Calatola uxpanapensis P. Vera \& T. Wendt, Acta Bot. Mex. 54:40, fig. 1. 2001. Observación: A. Molina R. 8204 (F). 


\section{Iridaceae}

*Sisyrinchium tenuifolium Humb. \& Bonpl. ex Willd., Enum. pl. 2:691. 1809. Observación: G. Davidse, M. Sousa \& J.L. Linares 35150 (MO), J.R. Martínez \& C. Bejarano 124 (MO, TEFH?).

\section{Lamiaceae (Labiatae)}

**Clerodendrum chinense (Osbeck) Mabb., Pl.-Book:707. 1989. Observación: C. Nelson \& G. Cruz 9185 (MO, TEFH), C. Nelson, E. Vargas, M. Erazo, M. García \& M. Sierra 2853 (MO, TEFH), G. Yong 305 (MO). Citada en Nelson Sutherland (2008) como Clerodendrum philippinense Schauer.

*Clinopodium ludens (Shinners) A. Pool, Novon 18(4):509. 2008. Observación: A. Molina R. 3020 (F).

*Cunila leucantha Kunth ex Schltdl. \& Cham., Linnaea 6:373. 1831. Observación: A. Molina R. 11351 (US).

*Plectranthus verticillatus (L.f.) Druce, Rep. Bot. Soc. Exch. Club Brit. Isles 4. Suppl. 2: 640. 1917. Observación: A. Molina R. 34047 (MO).

*Salvia colonica Standl. \& L.O. Williams ex Klitg., Novon 17(2):206, fig. 1 p-x. 2007. Observación: A. Molina R. \& A.R. Molina 24589 (F), tipo; isotipo (MO). El tipo fue colectado en el departamento de Choluteca.

**Salvia opertiflora Epling, Bull. Torrey Bot. Club 68(8):568. 1941. Observación: A. Molina R. \& A.R. Molina 25483 (MO). Citada en Nelson Sutherland (2008) como Salvia alariformis L.O. Williams.

*Salvia recurva Benth., Prodr. 12:336. 1848. Observación: R.J. Evans 1135 (MO, TEFH).

*Salvia rubiginosa Benth. in DC., Prodr. 12: 301. 1848. Observación: A. Molina R. 20305 (F).

*Scutellaria aurantiaca A. Pool, Novon 16(3):398, fig. 3B (2006). Observación: J.G. Saunders 1114 (NY), tipo. Pool (2006) describe esta especie como endémica de Honduras.

*Scutellaria dumetorum Schltdl., Linnaea 7:400. 1832. Observación: G. Davidse, M. Sousa \& J.L. Linares 35236 (EAP, MO).

*Scutellaria molinarum A. Pool, Novon 16(3):395, fig. 3A. 2006. Observación: W.G. D'Arcy 17862 (EAP, MO), A. Molina R. \& A.R. Molina 27861 (EAP, F), A. Molina R., L.O. Williams, A.R. Molina, T.P. Williams \& J.A. Molina 31274 (EAP).

*Tetradenia riparia (Hochst.) Codd, Bothalia 14:181. 1983. Observación: A. Molina R. 34130 (MO). Nombre común: mirro.

*Teucrium canadense L., Sp. pl. 2: 564. 1753. Observación: A. Molina R. 517 (MO).

\section{Lauraceae}

*Cinnamomum brenesii (Standl.) Kosterm., Reinwardtia 6(1):20. 1961. Observación: J.G. Saunders 529 (MO).

*Ocotea atlantica van der Werff, Novon 11(4):504, fig. 2. 2001. Observación: G. Davidse, R. Zúñiga \& P.R. House 34505 (MO), TEFH), R.J. Evans 1064 (MO, TEFH), 1065 (MO, TEFH), R. Liesner 26149 (MO, TEFH), 26174 (MO, TEFH), C. Nelson, R. Andino \& D.J. Hernández 19124 (TEFH).

*Ocotea macrophylla Kunth, Nov. gen. sp., ed. quarto, 2:131. 1818. Observación: A. Molina R. 8138 (MO), C. Nelson \& E. Vargas 2419 (TEFH).

*Ocotea oblonga (Meisn.) Mez, Jahrb. König. Bot. Gart. Berlin 5:367. 1889. Observación: A. Molina R. 7197 (MO).

\section{Loganiaceae}

*Spigelia hamelioides Kunth, Nov. gen. sp., ed. quarto, 3:185. 1818 [1819]. Observación: C. Nelson 8333 (TEFH, US).

\section{Loranthaceae}

**Passovia pyrifolia (Kunth) Thiegh., Bull. Soc. Bot. France 42:172. 1895. Observación: T. Hawkins \& M. Merello 853 (MO), C. Nelson 3129 (MO, TEFH). Citada como Phthirusa pyrifolia (Kunth) Eichler en Nelson Sutherland (2008).

\section{Lecythidaceae}

*Eschweilera aguilarii S.A. Mori, Monogr. Syst. Bot. Missouri Bot. Gard. 111:903. 2007. Observación: G. Davidse, R. Zúñiga \& P.R. House 34523 (EAP, MO, NY). Endémica de Costa Rica y Honduras.

\section{Malpighiaceae}

*Mascagnia tomentosa C.E. Anderson, Brittonia 53(3):411, fig. 2. 2001. Observación: A. Molina R. 25944 (MO). Nombre común: mariposa.

*Tetrapterys tinifolia Triana \& Planch., Ann. Sci. Nat., Bot., sér. 4, 18:336. 1862. Observación: A. Molina R. 3650 (EAP, MO).

\section{Malvaceae}

*Bakeridesia exalata D.M. Bates, Gentes Herb. 10(5):449, fig. 6E, 8G, 10. 1973. Observación: A. Molina R., L.O. 
Williams, A.R. Molina \& T.P. Williams 31253 (MO, WIS). *Dombeya x cayeuxii André, Rev. Hort. 69:545. 1897. Observación: L.E. Matamoros 84 (MO), A. Molina R. \& A.R. Molina 35122 (MO).

*Hibiscus acetosella Welw. ex Ficalho, Bol. Soc. Geogr. Lisboa, sér. 2, 9-10:608. 1881. Observación: A. Molina R. 1975 (NY).

*Sida hirsutissima Mill., Gard. Dict., ed. 8, no. 11. 1768. Observación: C. Nelson \& E. Vargas 5123 (MO, TEFH).

*Trichospermum galeottii (Turcz.) Kosterm., Reinwardtia 6(3):278. 1962. Observación: A. Molina R. \& A.R. Molina 30793 (MO).

**Waltheria operculata Rose, Contr. U.S. Natl. Herb. 5(4):183. 1899. Observación: P.C. Standley 24564 MO). Citada en Nelson Sutherland (2008) como Turnera valleana Standl. \& L.O. Williams.

\section{Marantaceae}

* Calathea carolineae H. Kenn., J. Bot. Res. Inst. Texas 6(1):55, fig. 1. 2012. Observación: D.L. Kelly 12033 (BM), tipo; isotipo (HEH), R. Fritch 14 (BM). Kennedy (2012) la publica como endémica de Honduras.

*Goeppertia matudae (H. Kenn. \& Ganders) Borchs. \& S. Suárez, Syst. Bot. 37(3):632. 2012. Observación: $H$. Kennedy \& D.E. Breedlove 1414 (MO), tipo; isotipos (BM, BRIT, CAS, DAV, EAP, GB, MICH, MO, TEFH, TEX, UBC, UCR), S. Blackmore \& M. Chorley 4065 (MO), R.J. Evans 1791 (MO), P.J.M. Maas \& A. Molina R. 1034 (MO), 33206 (MO, TEFH), J.M. MacDougal, P.R. House \& R. Zúñiga 3106 (MO), A. Ortega U. 320 (MO), L. Tróchez 26 (MO).

\section{Marcgraviaceae}

*Marcgravia mexicana Gilg, Bot. Jahrb. Syst. 25. Beibl. 60:26. 1898. Observación: R. Aguilar \& R.J. Evans 4063 (EAP, MO).

\section{Melastomataceae}

*Clidemia novemnervia (DC.) Triana, Trans. Linn. Soc. London 28(1):136.1871 [1872]. Observación: L. Zelaya 185 (CAS).

**Henriettea hondurensis (Wurdack) Penneys, Michelang., Judd \& Almeda, Syst. Bot. 35(4):797. 2010. Observación: A. Molina R. 7273 (MO). Citada en Nelson Sutherland (2008) como Henriettella hondurensis Wurdack.

**Henriettea strigosa Gleason, Publ. Carnegie Inst. Wash. 522:340. 1940. Observación: C. Nelson \& E. Romero 4157
(CAS, TEFH). Citada en Nelson Sutherland (2008) como sinónimo de Henriettella seemannii Naudin.

*Monochaetum alpestre Naudin, Ann. Sci. Nat., Bot., sér. 3, 4:50. 1845. Observación: T. Hawkins, P.R. House, B. Allen \& R.C. Moran 94 (MO).

*Tibouchina urvilleana (DC.) Cogn., Fl. bras. 14(3):358, tab. 84, fig. 2. 1885. Observación: I. González 71 (MO).

\section{Meliaceae}

*Guarea mexicana Coronado, Novon 16(4):465. 2006. Observación: L.O. Williams \& A. Molina R. 14655 (MO).

**Guarea microcarpa C. DC., Bull. Herb. Boissier, sér. 2, 5(5):420. 1905. Observación: J.B. Edwards 410 (F). Citada como sinónimo de Guarea glabra Vahl en Nelson Sutherland (2008).

**Guarea tonduzii C. DC., Smithsonian Misc. Collect. 68(6):4. 1917. Observación: R.J. Evans 1581 (MO). Citada en Nelson Sutherland (2008) como sinónimo de Guarea glabra Vahl.

\section{Menispermaceae}

*Odontocarya tamoides (Willd. ex DC.) Miers var. canescens (Miers) Barneby, Mem. New York Bot. Gard. 20:91. 1970. Observación: A. Molina R. \& A.R. Molina 34328 (MO).

*Orthomene schomburgkii (Miers) Barneby \& Krukoff, Mem. New York Bot. Gard. 22:80. 1971. Observación: G. Davidse \& R. Zúñiga 16323 (MO).

\section{Moraceae}

*Ficus hartwegii (Miq.) Miq., Ann. Mus. Bot. LugdunoBatavi 3(10):299. 1867. Observación: J.M. MacDougal, P.R. House \& R. Zúñiga 3132 (MO).

\section{Myristicaceae}

*Virola elongata (Benth.) Warb., Ver. Deutsch. Bot. Ges. 13:89. 1895 [1896]. Observación: P.R. House 2308 (MO).

\section{Myrtaceae}

**Calyptranthes lucida Mart. ex DC., Prodr. 3:258. 1828. Observación: J.G. Saunders 721 (F). Citada en Nelson Sutherland (2008) como Calyptranthes pendula O. Berg.

*Eugenia gaumeri Standl., Publ. Field Mus. Nat. Hist., Bot. Ser. 8(1):28. 1930. Observación: J.B. Edwards 123 (F), A. Molina 7081 (F, GH). 
**Eugenia hiraeifolia Standl., Field Mus. Nat. Hist., Bot. Ser. 17:202. 1937. Observación: D.L. Hazlett 3253 (F). Citada en Nelson Sutherland (2008) como sinónimo de Eugenia salamensis Donn. Sm., especie que todavía no ha sido encontrada en Honduras.

**Eugenia tapirorum Standl., Publ. Field Mus. Nat. Hist., Bot. Ser. 9(4):321. 1940. Observación: T.G. Yuncker, J.M. Koepper \& K.A. Wagner 8700 (F), tipo; isotipos (BM, F, MICH, MO, NY), 8385 (BM, F, GH, MICH, MO, NY, UC), T.G. Yuncker 8816 (MO), J.L. Linares 1395. Citada en Nelson Sutherland (2008) como sinónimo de Eugenia bumelioides Standl.

*Myrceugenia lanceolata (Juss. ex J. St.-Hil.) Kausel, Lilloa 13:135. 1947 [1948]. Observación: A. Molina R. 31734 (MO). Nombre común: grumichama.

**Myrcia amazonica DC., Prodr. 3:250. 1828. Observación: Von Hagen \& von Hagen 1375 (NY), 1411 (F). Citada en Nelson Sutherland (2008) como sinónimo de Myrcia leptoclada DC.

*Plinia trunciflora (O. Berg) Kausel, Ark. Bot. 3:507. 1956. Observación: A. Molina R. 14122 (F), 31477 (MO). Nombre común: jaboticaba.

**Psidium oligospermum DC., Prodr. 3:236. 1828. Observación: C. Nelson 4792 (MO, TEFH), L.O. Williams \& A. Molina R. 14143 (GH, MO). Citada en Nelson Sutherland (2008) como Psidium sartorianm (O. Berg) Nied. Para algunos botánicos esta especie es sinónimo de Eugenia molinae Barrie.

\section{Nyctaginaceae}

**Pisonia macranthocarpa (Donn. Sm.) Donn. Sm., Bot. Gaz. 20(7):293. 1895. Observación: A. Molina R. 22486 (MO), L.O. Williams \& A. Molina R. 10445 (MO). Citada en Nelson Sutherland (2008) como sinónimo de Pisonia aculeata $\mathrm{L}$.

\section{Ochnaceae}

**Ouratea pyramidalis L. Riley, Bull. Misc. Inform. Kew 1924(3):107. 1924. Observación: C. Nelson 2903 (MO, TEFH). Citada en Nelson Sutherland (2008) como sinónimo de Ouratea insulae L. Riley.

\section{Olacaceae}

**Hondurodendron urceolatum C. Ulloa, Nickrent, Whitef. \& D.L. Kelly, Ann. Missouri Bot. Gard. 97(3):459, fig. 2-3. 2010. Observación: D.L. Kelly, R. Fritch, D. Brady, C. Whitefoord \& M. Ramírez BC/3/MS 233 (TEFH), tipo; isotipos (BM, MO, TCD), $R$. Fritch, F. Laines \& $R$. Fernández GU/1/MS/ 869 (BM, MO), D.L. Kelly, K. Fagan \& P. Cortés 12036 (MO), D.L. Kelly \& A. Fiallos DA/2/MS 313 BM, HEH, MO), D.L. Kelly \& P. Ponce 12075 (MO). Esta planta fue descrita originalmente por Ulloa Ulloa et al. (2010) como endémica de Honduras en la familia de las Aptandraceae.

\section{Oleaceae}

*Jasminum laurifolium Roxb. fma. nitidum (Skan) P.S. Green, Kew Bull. 39(3):656. 1984. Observación: A. Molina R. 34051 (MO).

*Ligustrum ovalifolium Hassk., Cat. hort. bot. bogor.:119. 1844. Observación: A. Molina R. \& A.R. Molina 26071 (F).

\section{Orchidaceae}

**Acianthera pubescens (Lindl.) Pridgeon \& M.W. Chase, Lindleyana 16(4):245. 2001. Observación: A. Molina R. \& A.R. Molina 31145 (MO). Citada en Nelson Sutherland (2008) como sinónimo de Pleurothallis pubescens Lindl.

*Acronia hawkinsii Luer \& Endara, Monogr. Syst. Bot. Missouri Bot. Gard. 103:141, fig. 98. 2005. Observación: T. Hawkins, J. Montesinos \& P. Holm 126 (MO), tipo; isotipo (EAP). Luer y Andara (2005) describen esta especie como endémica de Honduras.

*Bulbophyllum sordidum Lindl., Edwards's Bot. Reg. 26. Misc.: 89. 1840. Observación: J.B. Edwards 264 (AMES).

*Camaridium hagsaterianum (Soto Arenas) M.A. Blanco, Lankesteriana 7(3):520. 2007. Observación: J.B. Edwards 82 (AMES).

*Coryanthes picturata Rchb. f., Bot. Zeitung (Berlin) 22(43):332. 1864. Observación: J.B. Edwards 649 (AMES).

**Cuitlauzina egertonii (Lindl.) Dressler \& N.H. Williams, Selbyana 24(1): 44. 2003. Observación: J.B. Edwards 345 (AMES). Citado en Nelson Sutherland (2008) como Osmoglossum egertoni Lindl.

*Dichaea acroblephara Schltr., Repert. Spec. Nov. Regni Veg. Beih. 19:71. 1923. Observvación: J.B. Edwards 94 (AMES).

**Didactylus oscitans (Ames) Luer, Monogr. Syst. Bot. Missouri Bot. Gard. 95:257. 2004. Observación: J.B. Edwards 515 (AMES). Citada en Nelson Sutherland como Pleurothallis oscitans Ames.

**Domingoa purpurea (Lindl.) Van den Berg \& Soto Arenas, Neodiversity 2:8. 2007. Observación: J.B. Edwards 157 (AMES). Citada en Nelson Sutherland (2008) como Nageliella purpurea (Lindl.) L.O. Williams.

**Effusiella immersa (Linden \& Rchb. f.) Luer, Monogr. 
Syst. Bot. Missouri Bot. Gard. 112:106. 2007. Observación: J.B. Edwards 354 (AMES). Citada en Nelson Sutherland (2008) como Pleurothallis immersa Linden \& Rchb. f.

**Encyclia insidiosa (Rchb. f.) Schltr., Repert. Spec. Nov. Regni Veg. Beih. 7:254. 1920. Observación: G. Davidse, M. Sousa \& J.L. Linares 35090 (MO). Citada en Nelson Sutherland (2008) como Encyclia diota (L.) Schltr. subsp. diota.

*Epidendrum glumarum Hamer \& Garay, Icon. Pl. Trop. 13: tab. 1219. 1985. Observación: L.O. Williams \& A. Molina R. 11143 (F, MO).

**Epidendrum isthmi Schltr., Repert. Spec. Nov. Regni Veg. Beih. 17:34. 1922. Observación: J.B. Edwards 244 (AMES). Citado como sinónimo de Epidendrum paniculatum Ruiz \& Pav. en Nelson Sutherland (2008).

*Epidendrum melistagum Hágsater, Orquídea (Mexico City), n. s., 11:28, fig. s. n. 1988. Observación: A. Molina R. 22227 (F, MO, NY).

*Epidendrum wendtii Hágsater \& Salazar, Icones Orchid. (México) 3: tab. 399. 1999. Observaciones: T. Hawkins 635 (MO).

*Epidendrum yojoaense Hágsater \& L. Sánchez S., Icon. Orchid. (Mexico) 7(4): tab. 800. 2004. Observación: J.B. Edwards 243 (AMES, MO). Hágsater y Sánchez S. (2004) la citan como endémica de Honduras.

*Galeandra arundinis Garay \& G.A. Romero, Vanishing Beauty: Native Costa Rican Orchids 1: 326, pl. 3. 2005. Observación: J.B. Edwards 491 (AMES).

*Govenia matudae E.W. Greenw. \& Soto Arenas, Icon. Orchid (México) 5-6: tab. 590. 2002 [2003]. Observación: T. Hawkins, P.R. House \& D. Mejía 534 (EAP, MO).

*Govenia quadriplicata Rchb. f., Beitr. Orchid.-K. C. Amer.: 75. 1866. Observación: J.B. Edwards 489 (AMES).

*Habenaria brownelliana Catling, Brittonia 39(3):402. 1987. Observación: J.G. Saunders 831 (F, LL, MO, TEFH), 910 (F, SEL).

**Habenaria pringlei B.L. Rob., Proc. Amer. Acad. Arts 27:184. 1892; vide Proc. Amer. Acad. Arts, n.s., 19:184. 1893. Observación: P.H. Allen 6584 (SEL), S. Blackmore \& M. Chorley 3983 (MO), J.M. MacDougal, P.R. House \& R. Zúñiga 3095 (MO). Citada en Nelson Sutherland (2008) como sinónimo de Habenaria bractescens Lindl.

*Isochilus alatus Schltr., Repert. Spec. Nov. Regni Veg. 10(254-256): 360. 1912. Observación: T. Hawkins 424 (AMO, EAP, MO, TEFH).

*Kraenzlinella erinacea (Rchb. f.) Solano, Icon. Orchid. (México) 5-6: xi. 2002 [2003]. Observación: J.B. Edwards 343 (AMES).

*Lophiaris carthagenensis (Jacq.) Braem, Schlechteriana 4 (1-2):17. 1993. Observación: A.H. Gentry et al. 7548 (MO).
*Maxillaria aciantha Rchb. f., Bot. Zeitung (Berlin) 10(49):858. 1852. Observación: J.B. Edwards 587 (MO), L.O. Williams \& A. Molina R. 11380 (MO).

*Maxillaria brunnea Linden \& Rchb. f., Bonplandia (Hannover) 2(23):281. 1854. Observación: J.B. Edwards 621 (AMES, MO).

*Maxillaria hagsateriana Soto Arenas, Orquídea (Mexico City), n.s., 12(2):252, fig. 1-4.1992. Observación: J.B. Edwards 82 (AMES).

*Maxillaria moralesii Carnevali \& J.T. Atwood, Lindleyana 11(1):31, fig. 2. 1996. Observación: J.B. Edwards 289 (AMES).

*Maxillaria porrecta Lindl., Bot. Reg. 24. Misc.:92. 1838. Observación: J.B. Edwards 621 (AMES, MO).

*Mesadenella petenensis (L.O. Williams) Garay, Bot. Mus. Leafl. 28(4):335. 1982. Observación: C. Thieme 5549 (BR).

**Microchilus lunifer (Schltr.) Ormerod, Lindleyana 17(4):217. 2002. Observación: A. Molina R. 8107 (MO). Citado en Nelson Sutherland (2008) como Erythrodes lunifera (Schltr.) Ames.

*Muscarella marginata (Rich.) Luer, Monogr. Syst. Bot. Missouri Bot. Gard. 105:111, fig. 75. 2006. Observación: J.B. Edwards 234A (AMES).

**Nemaconia striata (Lindl.) Van den Berg, Salazar \& Soto Arenas, Neodiversity 2(1):8. 2007. Observación: J.B. Edwards 394 (AMES). Citada en Nelson Sutherland como Ponera striata Lindl.

Octomeria yauaperyensis Barb. Rodr., Vellosia (ed. 2) 1: 122, tab. 837. 1891. Observación: J.B. Edwards 304 (AMES).

**Oestlundia luteorosea (A. Rich. \& Galeotti) W.E. Higgins, Selbyana 22(1):4. 2001. Observación: J.B. Edwards 137 (MO). Citada en Nelson Sutherland (2008) como Encyclia luteorosea (A. Rich. \& Galeotti) Dressler \& G.E. Pollard.

*Oncidium oerstedii Rchb. f., Bonplandia (Hannover) 2(7):91. 1854. Observación: J.B. Edwards 190 (AMES), 478 (AMES, US).

*Oncidium oliganthum (Rchb. f.) L.O. Williams ex Correll, Lloydia 10(4):212. 1948. Observación: T. Hawkins 547 (EAP, MO).

**Panmorphia barbulata (Lindl.) Luer, Monogr. Syst. Bot. Missouri Bot. Gard. 105:146, fig. 102a-b. 2006. Observación: J.B. Edwards 195 (AMES). Citada en Nelson Sutherland (2008) como Pleurothallis barbulata Lindl.

*Phragmipedium exstaminodium Castaño, Hágsater \& Ag. León, Orquídea (Mexico City), n.s., 9(2): 193.1984. Observación: A. Molina R. 3152 (EAP).

*Pleurothallis acrisepala Ames \& C. Schweinf., Sched. Orch. 8:22. 1925. Observación: J.M. MacDougal, P.R. 
House \& R. Zúñiga 3346 (MO).

*Pleurothallis discoidea Lindl., Edwards's Bot. Reg. 21: sub tab. 1797. 1835. Observación: T. Hawkins, P.R. House, B. Allen \& R.C. Moran 58 (MO).

*Pleurothallis sicaria Lindl., Edwards's Bot. Reg. 27. Misc.: 91. 1841. Observación: T. Hawkins \& M. Merello 778 (MO).

*Ponthieva trilobata (L.O. Williams) L.O. Williams, Fieldiana, Bot. 34(8):115. 1972. Observación: J.B. Edwards 282 (AMES).

*Pseudostelis spiralis (Lindl.) Schltr., Anexos Mem. Inst. Butantan, Secc. Bot. 1(4):38. 1922. Observación: A. Molina R. 11956 (F). Pleurothallis deregularis (Barb. Rodr.) Luer es sinónimo.

*Psygmorchis crista-galli (Rchb. f.) Dressler \& N.H. Williams, Nat. Ecuad. Orch. 4:883. 2003. Observación: J.B. Edwards 100 (AMES).

*Scaphyglottis imbricata (Lindl.) Dressler, Lankesteriana 3:28. 2002. Observación: A. Molina R. 5730 (F).

Sobralia amabilis (Rchb. f.) L.O. Williams, Ann. Missouri Bot. Gard. 33(1):30. 1946. Observación: L.O. Williams \& A. Molina R. 12777 (F).

*Sobralia warszewiczii Rchb. f., Bot. Zeitung (Berlin) 10:714. 1852. Observación: T. Hawkins 928 (MO).

*Stanhopea dodsoniana Salazar \& Soto Arenas, Lindleyana 16(3):144, fig. 1, 2B. 2001. Observación: J.B. Edwards 52 (US).

**Stenorrhynchos aurantiacum (La Llave \& Lex.) Lindl., Gen. Sp. Orchid. Pl.:479. 1840. Observación: G. Davidse, M. Sousa \& J.L. Linares 35466 (MO), A. Molina R. 412 (MO), L.O. Williams \& A. Molina R. 10153 (MO). Citado en Nelson Sutherland (2008) como sinónimo de Dichromanthus aurantiacus (La Llave \& Lex.) Salazar \& Soto Arenas.

*Stenorrhynchos glicensteinii Christenson, J. Orchideenfreund 12(1):20. 2005. Observación: T. Hawkins, P.R. House \& D. Mejía 171 (EAP, MO, TEFH).

**Stenotyla lendyana (Rchb. f.) Dressler, Lankesteriana 5(2):96. 2005. Observación: Citada como Chondrorrhyncha lendyana Rchb. f. en Nelson Sutherland 2008).

**Trichocentrum aurisasinorum (Standl. \& L.O. Williams) M.W. Chase \& N.H. Williams, Lindleyana 16(2):137. 2001. Observación: A. Molina R. 1823 (MO). Citada en Nelson Sutherland (2008) como Oncidium aurisasinorum Standl. \& L.O. Williams.

**Trichocentrum lindenii (Brongn.) M.W. Chase \& N.H. Williams, Lindleyana 16(2):137. 2001. Observación: J.B. Edwards 99 (AMES). Citada en Nelson Sutherland (2008) como Oncidium lindenii Brongn.

*Trichocentrum luridum (Lindl.) M.W. Chase \& N.H.
Williams, Lindleyana 16(2):137. 2001. Observación: E.S. Bennett s.n. (AMES), J.B. Edwards 384 (AMES), G.G. Stewart s.n. (AMES).

*Trichocentrum microchilum (Bateman ex Lindl.) M.W. Chase \& N.H. Williams, Lindleyana 16(2):138. 2001. Observación: A. Molina R. 22431 (MO, SEL).

*Trichocentrum undulatum (Sw.) Ackerman \& M.W. Chase, Lindleyana 16(4):225. 2001. Observación: J.B. Edwards 710 (AMES), S. Blackmore \& G.L.A. Heath 1955 (MO).

\section{Orobanchaceae}

*Agalinis marítima (Raf.) Raf., New fl. 2:62. 1836 [1837]. Observación: C. Nelson 4817 (MO, TEFH), C. Nelson \& E. Vargas 760 (MO, TEFH), C. Nelson \& E. Vargas 4970 (MO, TEFH). Género citado en Nelson Sutherland (2008) en la familia de las Scrophulariaceae.

**Castilleja integrifolia L. f. var. alpigena L.O. Williams, Fieldiana, Bot. 34(8):119. 1972. Observación: B.K. Holst 1222 (MO). Género citado en Nelson Sutherland (2008) en la familia de las Scrophulariaceae.

\section{Oxalidaceae}

*Oxalis microcarpa Benth., Pl. hartw.: 115. 1839. Observación: A. Molina R. 10938 (F).

\section{Phyllanthaceae}

**Phyllanthus grandifolius L., Sp. pl. 2:981. 1753. Observación: Citada en Nelson Sutherland (2008) como Phyllanthus glaucescens Kunth en la familia de las Euphorbiaceae.

*Phyllanthus nutans Sw., Prodr.:27. 1788. Observación: G.R. Proctor 32542 (IJ).

\section{Phytolaccaceae}

*Phytolacca octandra L., Sp. pl. ed. 2, 1: 631. 1762. Observación: R.J. Evans 1274 (MO).

\section{Picrodendraceae}

*Picrodendron baccatum (L.) Krug \& Urb. ex Urb., Bot. Jahrb. Syst. 15:308. 1892. Observación: Citado en Nelson Sutherland (2008) en la familia de las Euphorbiaceae.

\section{Piperaceae}


**Peperomia conocarpa Trel., Field Mus. Nat. Hist., Bot. Ser. 17(4):332. 1938. Observación: D. Mejía 487 (EAP, MO, TEFH). Citada en Nelson Sutherland (2008) como sinónimo de Peperomia quadrifolia (L.) Kunth.

*Peperomia donaguiana C. DC., Linnaea 37:382. 1872. Observación: D. Dey \& E. Siegerist 284 (MO).

**Peperomia praeteruentifolia Trel., Publ. Field Mus. Nat. Hist., Bot. Ser. 17(4):337. 1938. Observación: T.G. Yuncker, R.F. Dawson \& H.R. Youse 5614 (ILL), tipo, (MO, F), isotipos. Citada en Nelson Sutherland (2008) como sinónimo de Peperomia sancarlosiana C. DC.

**Piper arieianum C. DC., Prim. fl. costaric. 2(3):214. 1899. Observación: A.F. Clewell 4235 (MO). Citada como sinónimo de Piper trigonum C. DC. en Nelson Sutherland (2008).

Piper picobonitoense F.G. Coe \& Bornst., Syst. Bot. 34(3):492, fig. 1. 2009. Observación: A.J. Bornstein \& F.G. Coe 942 (NY), tipo; isotipos (COON, F, MO, SEMO, TEFH), R. Liesner 26084 (MO), 26269 (MO). Coe \& Bornstein (2009) describen esta especie como endémica de Honduras.

\section{Plantaginaceae}

**Achetaria scutellarioides (Benth.) Kuntze, Rev. gen. pl. 2:456. 1891. Observación: C. Nelson \& E. Vargas 4982 (TEFH). Citada en Nelson Sutherland (2008) como Veronica arvensis L. en la familia de las Scrophulariaceae.

**Bacopa bacopoides (Benth.) Pulle, Enum. Pl. Surinam: 415. 1906. Observación: Proctor 38815 (BM). Género citado en Nelson Sutherland (2008) en la familia de las Scrophulariaceae.

*Bacopa egensis (Poepp.) Pennell, Proc. Acad. Nat. Sci. Philadelphia 98(2):96. 1946. Observación: Davidse \& Pohl 2417 (MO).

**Callitriche terrestris Rafin., Med. Repos. New York 5:358. 1808. Observación: P.C. Standley \& A. Molina R. 4250 (F). Citada en Nelson Sutherland (2008) como Callitriche deflexa A. Braun ex Hegelm. en la familia de las Callitrichaceae cuyos taxones han sido trasladados a la familia de las Plantaginaceae.

**Cymbalaria muralis P. Gaertn., B. Mey. \& Scherb., Oekon. Fl. Wetterau 2:397. 1800. Observación: N. Castro 136 (MO). Citada en Nelson Sutherland (2008) en la familia de las Scrophulariaceae.

*Russelia standleyi Carlson, Fieldiana, Bot. 29(4):257. 1957, Observación: A. Molina R. \& A.R. Molina 27862 (F). El género Russelia Jacq. y todas sus especies son citados en Nelson Sutherland (2008) en la familia de las Scrophulariaceae.
**Schistophragma mexicanum Benth. ex Endl., Syn. pl. 3:513. 1843. Observación: J.L. Linares, E.Y. Guerrero \& P.S. Flores 6651 (MO). Citada en Nelson Sutherland (2008) como Schistophragma pusillum Benth. en la familia de las Scrophulariaceae.

*Tetranema michaelfayanum Christenh., Phytotaxa 14:57. 2010. Observación: R.J. Evans 1354 (BM) tipo; isotipo (MO). Dedicada a un botánico ajeno a la flora de Honduras. Christenhusz (2010) publicó esta especie como endémica de Honduras

**Veronica $x$ andersonii Lindl. \& Paxton, Paxt. Fl. Gard. 2:3. 1853. Observación: A. Molina R. 34044 (MO). Citada en Nelson Sutherland (2008) como Hebe $x$ andersonii (Lindl. \& Paxton) Cockayne. Nombre común: verónica.

\section{Poaceae (Gramineae)}

*Muhlenbergia lehmanniana Henrard, Meded. Rijks-Herb. 40:59. 1921. Observación: C. Nelson, A.L. Díaz, R. Rodríguez, R. Andino, H.A. Martínez \& E. Romero 6818 (MO, TEFH).

**Olmeca clarkiae (Davidse \& R.W. Pohl) Ruiz-Sánchez \& Mejía-Saulés, Taxon 60(1):93. 2011. Observación: $G$.

Davidse \& R. Zúñiga 34651 (MO). Citado en Nelson

Sutherland (2008) como Aulonemia clarkiae Davidse \&

R.W. Pohl.

*Urochloa ruziziensis (R. Germ. \& C.M. Evrard) Crins, J. Arnold Arbor. Suppl. Ser. 1:269. 1991. Observación: A. Molina R. 31786 (MO). Nombre común: pasto bandera. **Zuloagaea bulbosa (Kunth) Bess, Syst. Bot. 31(4):666. 2006. Observación: A. Molina R. 348 (MO, US). Citado como Panicum bulbosum Kunth en Nelson Sutherland (2008).

\section{Podocarpaceae}

*Nageia nagi (Thunb.) Kuntze, Revis. gen. pl. 2:798. 1891. Observación: A. Molina R. 33937 (MO), 34089 (MO), 34349 (MO).

*Podocarpus macrostachys Parl., Prodr. 16(2):510. 1868 Observación: T. Hawkins, B. Allen \& R.J. Evans 587 (MO).

\section{Podostemaceae}

*Marathrum utile Tul., Ann. Sci. Nat., Bot., sér. 3, 11:95. 1849. Observación: P.J.M. Maas, H. Maas, R. Zúñiga \& T. Gómez 8495 (EAP).

\section{Polemoniaceae}


*Cobaea triflora Donn. Sm., Bot. Gaz. 13(4):75. 1888. Observación: T. Hawkins 190 (EAP, MO, TEFH), 234 (EAP, MO, TEFH).

\section{Polygalaceae}

*Polygala linoides Poir. var. laxiflora Grondona, Darwiniana 8(2-3):347. 1948. Observación: L.O. Williams \& A. Molina R. 15936 (MO).

*Polygala scoparia Kunth, Nov. gen. sp., ed. quarto, 5:399. 1821 [1823]. Observación: E.G. Seler 3345 (MO), L.O. Williams \& A. Molina R. 10693 (MO).

\section{Primulaceae}

**Ardisia copeyana Standl., Publ. Field Mus. Nat. Hist., Bot. Ser. 18(3):886. 1938. Observación: C. Nelson, J. Escobar \& R. Andino 8035 (TEFH). Nelson Sutherland (2008) cita a Ardisia standleyana P.H. Allen, la cual es sinónimo de A. copeyana Standl. Nombre común: uvilla.

**Samolus floribundus Kunth, Nov. gen. sp., ed. quarto, 2:224. 1819. Observación: S. Blackmore \& M. Chorley 4015 (BM), R. Rodríguez 189 (MO). Citada en Nelson Sutherland como Samolus valerandi $\mathrm{L}$.

\section{Ranunculaceae}

**Clematis grossa Benth., Pl. hartw.: 33. 1840. Observación: J.B. Edwards P-728 (MO), R. J. Evans 1211 (MO, TEFH). Citado en Nelson Sutherland (2008) como sinónimo de Clematis haenkeana C. Presl.

*Consolida ajacis (L.) Schur, Verh. Mitth. Siebenburg. Vereins Naturwiss. Hermannstadt 4(3):47. 1853. Observación: J. Valerio 3154 (F).

*Ranunculus petiolaris Kunth ex DC., Syst. nat. 1:287. 1817 [1818]. Observación: G. Davidse, M. Sousa \& J.L. Linares 35222 (MO).

\section{Rosaceae}

*Fragaria x ananassa Duchesne ex Rozier, Cours compl. agric. 5:52, tab. 5, fig. 1. 1784 [1785]. Observación: $R$. Matamoros 42 (MO), A. Molina R. 34003 (MO).

**Lachemilla pectinata (Kunth) Rothm., Repert. Spec. Nov. Regni Veg. 42(1081-1085):171. 1937. Observación: $T$. Hawkins \& B. Allen 314 (EAP, MO, TEFH). Citada como Alchemilla punctata Kunth in Nelson Sutherland (2008).

*Photinia microcarpa Standl. var. hintonii J.B. Phipps, Canad. J. Bot. 70(11):2154. 1992. Observación: R.J. Evans 1733 (MEXU, MO), D. Mejía 199 (EAP, MO, TEFH).
**Prunus serotina Ehrh., Gartenkalender 3:285. 1783. Observación: G. Davidse, M. Sousa \& J.L. Linares 35230 (MO). Citada en Nelson Sutherland (2008) como Prunus capuli Cav.

*Rosa minutifolia Engelm., Bull. Torrey Bot. Club 9(8):97. 1882. Observación: M.M. García 44 (MO), A. Rubio G. 29 MO), M.E. Villeda 66 (MO).

\section{Rubiaceae}

*Arachnothryx chiriquiana (Lorence) Borhidi, Acta Bot. Hung. 40(1-4):15. 1996 [1997]. Observación: D.L. Hazlett, A. Brant \& R. Zúñiga 8018 (MO).

**Arachnothryx rufescens (B.L. Rob.) Borhidi, Acta Bot. Acad. Sci. Hung. 28:69. 1982. Observación: Citada como sinónimo de Arachnothryx gracilis (Hemsl.) Borhidi en Nelson Sutherland (2008).

*Chiococca belizensis Lundell, Amer. Midl. Naturalist 29:492. 1943. Observación: G. Davidse, M. Sousa \& J.L. Linares 35574 (MO).

**Chione venosa (Sw.) Urb., Symb. antill. 4(4):594. 1911. Observación: C. Nelson \& R. Andino 18157 (TEFH). Citado en Nelson Sutherland (2008) como Chione guatemalensis Standl. \& Steyerm.

*Coutaportla guatemalensis (Standl.) Lorence, Syst. Bot. 11(1): 210. 1986. Observación: J.G. Saunders 1118 (MO).

**Elaeagia mariae Wedd., Hist. nat. quinquinas:94. 1894. Observación: C. Nelson, J. Escobar \& R. Andino 7948 (MO, TEFH). Citada en Nelson Sutherland (2008) como Elaeagia karstenii Standl.

*Guettarda subcapitata C.M. Taylor, Novon 11(1):128, fig. 2. 2001. Observación: A. Molina R. \& A.R. Molina 31051 (MO), E. Romero 62 (MO).

*Hamelia magnifolia Wernham, J. Bot. 49(583):210. 1911. Observación: J.M. MacDougal, P.R. House \& R. Zúñiga 3318 (MO).

*Hoffmannia cauliflora Hemsl., Diagn. pl. nov. mexic. 2:30. 1879. Observación: T. Hawkins \& D. Mejía 703 (EAP, HEH, MO, TEFH).

**Hoffmannia excelsa (Kunth) K. Schum., Fl. bras. 6(6):327. 1889. Observación: T. Hawkins, D. Mejía \& B. Allen 739 (EAP, MO, TEFH), E.M. Martínez \& O. Téllez 12959 (MO). Citada en Nelson Sutherland (2008) como Hoffmannia montana L.O. Williams.

*Margaritopsis microdon (DC.) C.M. Taylor, Syst. Geogr. Pl. 75(2):169. 2005. Observación: C. Nelson 8297 (MO, TEFH).

*Markea panamensis Standl., J. Arnold Arbor. 11(2):127. 1930. Observación: A.E. Brant \& R. Zúñiga 2820 (MO).

**Palicourea tetragona (Donn. Sm.) C.M. Taylor \& 
Lorence, Novon 20(4):490. 2010. Observación: A.F. Clewell 4547 (MO). Citado como Psychotria chiapensis Standl. en Nelson Sutherland (2008).

*Randia lasiantha (Standl.) Standl., Contr. U.S. Natl. Herb. 20(6):201. 1917. Observación: J.G. Saunders 369 (MO).

**Sabicea villosa Willd. ex Roem. \& Schult. var. adpressa (Werham) Standl., Publ. Field Columb. Mus., Bot. Ser. 7(1):52. 1930. Observación: J.M. MacDougal, P.R. House $\&$ R. Zúñiga 3210 (MO). Citada en Nelson Sutherland (2008) como sinónimo de Sabicea villosa Willd. ex Roem. \& Schult.

*Sabicea villosa Willd. ex Roem. \& Schult. var. sellowii (Wernham) Steyerm., Mem. New York Bot. Gard. 17:314. 1967. Observación: C. Nelson \& G. Cruz 9239 (MO, TEFH).

**Spermacoce alata Aubl., Hist. pl. Guiane 1:60, tab. 22, fig. 7. 1775. Observación: L. Tróchez 176 (MO). Citada como Spermacoce latifolia Aubl. en Nelson Sutherland (2008)

*Spermacoce exilis (L.O. Williams) C.D. Adams, Fieldiana, Bot., n.s., 33:316, fig. 5. 1993. Observación: C. Nelson 759 (MO, TEFH).

\section{Rutaceae}

**Triphasia aurantiola Lour., Fl. cochinch. 1:153. 1790. Observación: T. Clare 32 (MO), A. Molina R., A.R. Molina \& J.A. Molina 32073 (MO), L. Zelaya 38 (MO). Citada en Nelson Sutherland (2008) como sinónimo de Triphasia trifolia (Burm. f.) P. Wilson.

**Zanthoxylum monophyllum (Lam.) P. Wilson, Bull. Torrey Bot. Club 37(2):86. 1910. Observación: G. Davidse, M. Sousa \& J.L. Linares 35384 (MO). Citada en Nelson Sutherland (2008) como Zanthoxylum schreberi (J.F. Gmel.) Reynel.

\section{Salicaceae}

*Samyda mexicana Rose, Contr. U.S. Natl. Herb. 5(4):199. 1899. Observación: A. Molina R. 3384 (MO).

**Xylosma cinerea (Clos) Hemsl., Biol. centr.-amer., Bot. 1(1): 57. 1879. Observación: C. Nelson, E. Romero, A. Rubio G. \& M. Pereira 4006 (TEFH). Citada en Nelson Sutherland (2008) como sinónimo de Xylosma flexuosa (Kunth) Hemsl. Nombres comunes: clavo, morro.

\section{Sapindaceae}

*Paullinia serjaniifolia Triana \& Planch., Ann. Sci. Nat., Bot. 18:356. 1862. Observación: A. Molina R. 5918 (MO),
8767 (MO).

*Paullinia turbacensis Kunth, Nov. gen. sp., ed. quarto, 5:114. 1821. Observación: D. Burch 6099 (MO).

\section{Scrophulariaceae}

**Bontia daphnoides L., Sp. pl. 2:638 [como 938]. 1753. Observación: C. Nelson, D. Ruiz \& S. Gómez 9746 (MO, TEFH), G.G. Nelson 76 (GH), G.R. Proctor 32507. Citada en Nelson Sutherland (2008) en la familia de las Myoporaceae.

**Buddleja euryphylla Standl. \& Steyerm., Publ. Field Mus. Nat. Hist., Bot. Ser. 23(5):223. 1947. Observación: Berendsohn et al. (2009) la citan de Honduras. El género Buddleja L. y todas sus especies son citadas en Nelson Sutherland (2008) en la familia de las Buddlejaceae.

**Capraria frutescens (Mill.) Britten, J. Bot. 45: 315. 1907. Observación: C. Nelson, A.L. Díaz, R. Rodríguez, R. Andino, H.A. Martínez \& E. Romero 6229 (MO, TEFH). Previamente identificada como Capraria biflora $\mathrm{L}$.

\section{Smilacaceae}

*Smilax officinalis Kunth, Nov. gen. sp., ed. quarto., 1:271. 1815 [1816]. Observación: J.G. Saunders 285 (MO).

\section{Solanaceae}

**Cestrum guatemalense Francey, Candollea 6:98. 1935. Observación: A. Molina R. 8774 (MO). Citado en Nelson Sutherland (2008) como Cestrum anagyris Dunal con el espécimen R. Keyser 1200 (TEFH).

*Datura discolor Bernh., Linnaea 8:138. 1833. Observación: G. Davidse, M. Sousa \& J.L. Linares 35511 (MO).

*Lycianthes inaequilatera (Rusby) Bitter, Lycianthes: 439. 1919. Observación: J.M. MacDougal, P.R. House \& R. Zúñiga 3169 (MO).

*Markea panamensis Standl., J. Arnold Arbor. 11(2):127. 1930. Observación: A. Brant \& R. Zúñiga 2820 (MO).

*Physalis leptophylla B.L. Rob. \& Greenm., Proc. Amer. Acad. Arts 29:389. 1894. Observación: A. Molina R. 24681 (F).

**Solanum deflexum Greenm., Proc. Amer. Acad. Arts 32(16):301. 1897. Observación: A.L. Díaz (MO). Citada en Nelson Sutherland (2008) como sinónimo de Solanum adscendens Sendtn.

**Solanum fortunense Bohs, Taxon 44(4):584. 1995. Observación: $R$. Evans 1586 (MO, TEFH). Citado en Nelson Sutherland (2008) como Cyphomandra 
dolichocarpa Bitter.

*Solanum pseudocapsicoides L., Sp. pl. 1:184. 1753. Observación: T. Clare 33 (MO).

*Solanum thelopodium Sendtn., Fl. bras. 10:46. 1846. Observación: C. Nelson \& M. Espinal 5336 (MO, TEFH).

*Solanum viarum Dunal in A. DC., Prodr. 13(1):240. 1852. Observación: "Una planta de $30 \mathrm{~cm}$ de altura y con frutos fue colectada el 26 de noviembre de 2007 en el estacionamiento del edificio de la carrera de Desarrollo Socio Económico y Ambiente (N 1400'36.5” O $\left.87^{\circ} 00^{\prime} 59.7^{\prime \prime}\right)$, en El Zamorano, Honduras. La identificación es del Dr. Richard Weaver del Department of Plant Industries, Florida Department of Agriculture and Consumer Services. El material fue depositado en la colección de plantas del Department of Plant Industries con código Rodrigo Díaz B-2007-782", según Díaz, Samayoa \& Overholt (2008).

*Solanum volubile Sw., Fl. Ind. Occid. 1:458. 1797. Observación: C. Nelson \& E. Vargas 5095 (MO, TEFH).

**Solanum yucatanum Standl., Publ. Field Mus. Nat. Hist., Bot. Ser. 8(1):443. 1930. Observación: Citada en Nelson Sutherland (2008) como sinónimo de Solanum dasyanthum Brandegee.

\section{Styracaceae}

*Styrax warszewiczii Perkins, Bot. Jahrb. Syst. 31(3):480. 1901. Observación: S. Lagos-Witte, C. Torres \& P.R. House 86 (MO), A. Molina R. 13 (MO).

\section{Symplocaceae}

*Symplocos pycnantha Hemsl., Biol. centr.-amer., Bot. 2(10):302. 1881. Observación: A. F. Clewell 3800 (MO), $T$. Hawkins, P.R. House \& D. Mejía 150 (MO), A. Molina R. \& A.R. Molina 25537 (MO), C. Nelson \& E. Romero 4324 (MO, TEFH), L. Zelaya 147 (MO).

\section{Talinaceae}

**Talinum fruticosum (L.) Juss., Gen. pl.: 312. 1789. Observación: G. Davidse, M. Sousa \& J.L. Linares 35508 (MO). Citada en Nelson Sutherland (2008) en la familia de las Portulacaceae.

\section{Theaceae}

**Gordonia brenesii (Standl.) Q. Jiménez, Phytoneuron 2011(10):2. 2011. Observación: P. Allen 6364 (MO), T. Hawkins 465 (EAP, MO, TEFH), D.L. Hazlett 3108 (MO),
N. Zamora V. 1742 (CR, MO). Citada en Nelson Sutherland (2008) como sinónimo de Gordonia brandegeei H. Keng con el nombre de Laplacea brenesii Standl.

\section{Thymelaeaceae}

**Daphnopsis ficina Standl. \& Steyerm., Publ. Field Mus. Nat. Hist., Bot. Ser. 22(4):254. 1940. Observación: Citada en Nelson Sutherland (2008) como Daphnopsis vicina Standl. \& Steyerm.

*Daphnopsis selerorum Gilg, Verh. Bot. Vereins Prov. Brandenburg 58:153. 1917. Observación: A.E. Brant \& D. Hazlett 2906 (BKL, MO, NY, US).

\section{Urticaceae}

*Pilea auriculata Liebm., Kongel. Danske Vidensk. Selsk. Skr., Naturvidensk. Math. Afd., ser. 5, 2:299.1851. Observación: A. Molina R., A.R. Molina \& J.A. Molina 32207 (MO). Nombre común: hierba de peña.

*Pilea depressa (Sw.) Blume, Mus. Bot. 2(3):46. 1856. Observación: A. Molina R. 34158 (MO).

*Pilea irrorata Donn. Sm., Bot. Gaz. 19(1):11. 1894. Observación: D.L. Hazlett \& A. Brant 8097 (MO).

*Urera laciniata Goudot ex Wedd., Ann. Sci. Nat., Bot., sér. 4, 18:203. 1854. Observación: J.G. Saunders 1204 (NY) *Urera verrucosa (Liebm.) V.W. Steinm., Acta Bot. Mex. 71:39. 2005. Observación: C. Nelson et al. 3955 (MO, TEFH).

\section{Valerianaceae}

**Valeriana candolleana Gardner, London J. Bot. 4:112. 1845. Observación: A.F. Clewell 3822 (MO), A.F. Clewell \& D.L. Hazlett 3894 (MO). Citada como Valeriana scandens L. subsp. candolleana (Gardner) K.A.E. Müll. en Nelson Sutherland (2008).

\section{Verbenaceae}

**Lippia bracteosa (M. Martens \& Galeotti) Moldenke, Phytologia 2(7):226. 1947. Observación: A. Molina R. \& A.R. Molina 24429 (NY), C. Nelson 2180 (MO, TEFH). Citada en Nelson Sutherland (2008) como Lippia controversa Moldenke.

**Verbena tenuisecta Briq., Annuaire Conserv. Jard. Bot. Genève 7-8:294. 1904. Observación: A. Molina R. \& A.R. Molina 34288 (MO), P.C. Standley 5147 (F). Citada en Nelson Sutherland (2008) como Glandularia tenuisecta (Briq.) Small. Nombre común: verbena. 


\section{Violaceae}

*Gloeospermum diversipetalum L.O. Williams, Ceiba 4(5):273. 1955. Observación: R. Zúñiga 438 (TEFH). El tipo, P.H. Allen 5278, que estaba en el herbario EAP, junto con otros tipos, fue transferido por L.O. Williams en 1956 al Herbario Nacional de los Estados Unidos (US) alegando mayor protección (Dorr et al. 2009). Una pérdida notable para EAP y un acto de expoliación por parte del señor Williams.

*Viola nannei Pol., Linnaea 41(5-6):547. 1877. Observación: R.J. Evans 1717 (MO).

\section{Vitaceae}

**Cissus ulmifolia (Baker) Planch., Monogr. phan. 5(2):552. 1887. Observación: J.M. MacDougal, P.R. House \& R. Zúñiga 3218 (MO, TEFH), 3392 (MO, TEFH). Cissus serrulatifolia L.O. Williams citada por Nelson Sutherland (2008) es sinónimo de Cissus ulmifolia (Baker) Planch.

\section{Ximeniaceae}

**Ximenia americana L., Sp. pl. 2:1193. 1753. Observación: J.R. Martínez 167 (MO), C. Nelson \& H.A. Martínez 7750 (MO, TEFH). Citada en Nelson Sutherland (2008) en la familia de las Olacaceae.

\section{Zamiaceae}

*Ceratozamia hondurensis J.L. Haynes, Whitelock, Schutzman \& R.S. Adams, Cycad Newslett. 31(2/3): 18, fig. 1-2. 2008. Observacion: A.E. Brant \& R. Zúñiga 2830 (EAP), tipo; isotipos (MEXU, MO, NY). Descrita de Atlántida, Honduras. Citada en Nelson Sutherland (2008) como Ceratozamia sp. (TEFH-39165, 39177, 39178, 39179, 39180, 40207). Publicada como endémica de Honduras por J.L. Haynes et al. (2008).

Zamia onan-reyesii C. Nelson \& G.G. Sandoval, Ceiba 49(1):135, fig. 1-6. 2008. Observación: O. Reyes 406 (TEFH), holotipo; J. Haynes, M. Bonta, G. Sandoval, C. O'Reilly, I. Zúniga \& J. Mendoza 45 (TEFH), paratipo, $R$ Alonzo \& A. Whittington June 2003, Whittington 200301 [sic] (FLAS), tipo de Zamia bussellii Schutzman et al. Nelson Sutherland \& Sandoval González (2008) describen esta especie como endémica de Honduras. En el mismo año, Schutzman et al. (2008) publicaron la especie Zamia bussellii, la que es considerada sinónimo de Zamia onanreyesii.

\section{Zygophyllaceae}

*Tribulus cistoides L., Sp. pl. 1: 387. 1753. Observación: A. Molina R. \& A.R. Molina 31529 (MO).

\section{Literatura Citada}

APG III. 2009. An update of the Angiosperm Phylogeny Group classification for the orders and families of flowering plants. Bot. J. Linn. Soc. 161:105-121.

Berendsohn, W.G., A.K. Gruber y J.A. Monterrosa Salomón. 2009. Nova Silva Cuscatlanica. Árboles nativos e introducidos de El Salvador. Parte 1: Angiospermae - Familias A a L. Englera 29(1):1-438.

Boggan, J.K. 2008. A review of the neotropical genera Amalophyllon, Niphaea, and Phinaea (GesneriaceaeGloxinieae). Selbyana 29(2):157-176.

Christenhusz, M.J.M. 2010. Nomenclatural corrections in Mesoamerican Plantaginaceae and a new species of Tetranema from Honduras. Phytotaxa 14:56-60.

Coe, F.G. y A.J. Bornstein. 2009. A new species of Piper (Piperaceae) from Cordillera Nombre de Dios, Honduras. Syst. Bot. 34(3):492-495.

Davidse, G., M. Sousa Sánchez, A.O. Chater, S. Knapp y F. Chiang Cabrera (eds.). 1994. Flora mesoamericana 6, 1, 4, 2, 3, 5, 7. Univ. Nac. Autón. México/Missouri Bot. Gard.

Díaz, R., A.C. Samayoa y W.A. Overholt. 2008. Solanum viarum Dunal (Solanaceae), primer reporte para Honduras. Ceiba 49(1):133-134.

Doblado Amador, L.S. 2011. Identificación y caracterización de tipos de bosque y su relación con variables ambientales en un paisaje fragmentado al norte de Honduras. Tesis M.Sc. CATIE. Turrialba, Costa Rica.

Dorr, L.J., G.E. Pilz y J.K. Boggan. 2009. The transfer of types from the Escuela Agrícola Panamericana to the U.S. National Herbarium in 1956 by Louis O. Williams. Taxon 58(3):993-1001.

Grayum, M.H. y A.P. Karney. 2012. A new Monstera species (Araceae) of economic importance from Honduras. Econ. Bot. 66(2):207-213.

Hágsater, E. y L. Sánchez S. 2004. The genus Epidendrum, Part 4. A fourth century of new species of Epidendrum. Icon. Orchid. (Mexico) 7: pl. 701-800.

Haynes, J.L., L.M. Whitelock, B.M. Schutzman y R.S. Adams. 2008. A new endemic Ceratozamia from Honduras (Cycadales: Zamiaceae). Cycad Newslett. 31(2/3):16-21. 
Henderson, A.J. 2005. A multivariate study of Calyptrogyne (Palmae). Syst. Bot. 30(1):60-83.

Hermann, F.J. 1949. Studies in Lonchocarpus and related genera, V: New species from Middle America and the Lonchocarpus guatemalensis complex. J. Wash. Acad. Sci. 39(9):306-313.

Kennedy, H. 2012. Calathea carolineae (Marantaceae), a new species endemic to Honduras. J. Bot. Inst. Texas 6(1):55-57.

Luer, C. y L. Endara in Luer. 2005. Icones Pleurothallidinarum XXVII: Dryadella and Acronia section Macrophyllae-Fasciculatae. Monogr. Syst. Bot. Missouri Bot. Gard. 103:1-310.

Nelson Sutherland, C.H. 2008. Catálogo de las plantas vasculares de Honduras. Espermatofitas. Tegucigalpa: Editorial Guaymuras.

Nelson Sutherland, C.H. y G.G. Sandoval González. 2008. Una especie nueva de Zamia (Zamiaceae) de Honduras. Ceiba 49(1):135-136.

Pool, A. 2006. New species of Scutellaria (Lamiaceae) from Mesoamerica. Novon 16(3):388-403.

Schatz, G.E. y P.J.M. Maas. 2010. Synoptic revisión of Stenanona (Annonaceae). Blumea 55(3):205-223.

Schutzman, B., R. Adams, J.L. Haynes y L.M. Whitelock (2008). A new endemic Zamia from Honduras (Cycadales: Zamiaceae). Cycad Newslett. 31(2/3):2226.
Shipunov, A.B. y E. Shipunova. 2011. Haptanthus story: Rediscovery of enigmatic flowering plant from Honduras. Amer. J. Bot. 98(4):761-763.

Sousa Sánchez, M. 2008. Las subespecies de Lonchocarpus rugosus Benth. (Leguminosae, Papilionoideae: Millettieae). Ceiba 49(1): 119-131.

Sousa Sánchez, M. 2009a. Standleyi, una nueva sección del género Lonchocarpus (Leguminosae), nuevas especies y subespecies para Mesoamérica y Sudamérica. Acta Bot. Mex. 86:39-69.

Sousa Sánchez, M. 2009b. La Sect. Punctati del género Lonchocarpus (Leguminosae, Papilionoideae, Millettieae) para Mesoamérica. Novon (19(2):239-255.

Stevens, W.D. 2005. Novelties in Cynanchum L., sensu Woodson, in Mesoamerica. Novon 15(4):620641.

Ulloa Ulloa, C., D.L. Nickrent, C. Whitefoord y D.L. Kelly 2010. Hondurodendron, a new monotypic genus of Aptandraceae from Honduras. Ann. Missouri Bot. Gard. 97(3):457-467.

Sometido para publicación el 5 de abril de 2013. Aceptado para publicación el 5 de junio de 2013. 